\title{
Exploring the potential of complex-vesicle based niosomal ocular system loaded with azithromycin: Development of in situ gel and ex vivo characterization
}

\author{
Nida Akhtar ${ }^{1}$, Rahul Kumar Singh ${ }^{1}$, Kamla Pathak $^{2 *}$ \\ ${ }^{1}$ Department of Pharmaceutics, Rajiv Academy for Pharmacy, P.O. Chhatikara, Mathura- 281001, Uttar Pradesh, India \\ ${ }^{2}$ Department of Pharmaceutics, Pharmacy College Saifai, Uttar Pradesh University of Medical Sciences, Saifai, Etawah-206130, Uttar Pradesh, India
}

Received: Feb13, 2017, Revised: Mar14, 2017, Accepted: Apr20, 2017

\begin{abstract}
Bacterial conjunctivitis characterized as pink eye referred as an inflammation of an eye caused by the enlargement of blood vessels present in conjunctiva, resulting in a red or bloodshot appearance of the eyes. Topical ocular delivery is found to be useful in treating conjunctivitis, but to maintain an effective drug concentration at a site of action in order to achieve desired pharmacological action is highly challenging. Thus, keeping in mind this limitation niosomal carrier was designed to provide localized drug delivery with enhanced residence time. Thus, the present investigation was targeted to explore the utility of niosomes loaded with azithromycin- $\beta-\mathrm{CD}$ complex. Azithromycin- $\beta-\mathrm{CD}$ complex was prepared and niosomes containing this complex were developed based on $3^{2}$ full factorial design using ether injection method and characterized. Optimized niosomal formulation (NF2) was selected on the basis of minimum vesicle size (306 $\pm 3.05 \mathrm{~nm})$, polydispersity index $(0.115 \pm 5.51)$, maximum zeta potential $(45.3 \pm 0.25 \mathrm{mv})$, entrapment efficiency $(78.17 \pm 1.81 \%)$ and \% CDR $(73.09 \pm 2.10)$. Optimized formulation was then formulated in the form of in situ gel (temperature sensitive) and evaluated. Optimized formulation [in situ gel (NG-5)] was found to exhibit superior in vitro drug release profile in comparison to Zithromax ${ }^{\circledR}$ eye drop. Better in-vitro mucoadhesive strength was observed and formulation was found to be non-irritant to the sclera surface. Thus, it can be put into conclusion that temperature-sensitive niosomal in situ ocular gel possessed increased residence time and provide localized drug delivery effective for the treatment of bacterial conjunctivitis.
\end{abstract}

Keywords: $\beta-\mathrm{CD}$, conjunctivitis, eye, penetration, residence time, vesicular carrier

Pharm Biomed Res 2017; 3(1): 22-33

\section{Introduction}

Azithromycin is commercially available as eye drops in a dose of $0.5 \% \mathrm{w} / \mathrm{v}$. The major limitation of the conventional formulations is enhanced precorneal drainage, because of which frequent instillation is needed. Use of high drug concentration is also required which usually leads to pulse kinetics pattern of drug release. The efficacy of azithromycin as an antibacterial agent in ophthalmic delivery is limited by poor solubility and limited permeability. Due to limited solubility and permeability, the ocular bioavailability gets reduced (1). A large portion of drug when administered using eye drops is reported to undergo loss by lachrymation, nasolachrymal drainage, tear turnover and tear dilution. These losses resulted in poor ocular bioavailability. The delivery of drug to ocular region basically depends upon the type of disease to be managed i.e. intraocular infection and extraocular infection. Extraocular related infection commonly includes conjunctivitis, keratoconjunctivitis, corneal ulcers, etc. Extraocular bacterial infection requires an antibiotic treatment regimen, as treatment with antibacterial agent reduces the chances of complications associated with chronic conditions such as corneal perforations if remain untreated (2).
The success of an antibacterial therapy depends upon achieving and maintaining the therapeutic drug concentration at optimum site of action for longer duration of time. Major limitation in maintaining extraocular infection is the lack of ability of the delivery system to provide long term therapeutic concentration in the extraocular region without compromising the intraocular region (3). The most suitable treatment of extraocular bacterial infection is topical therapy but has limited potential due to poor bioavailability at the site of action and due to constraints in ocular delivery (4). To conquer the problems associated with topical therapy of azithromycin, many novel delivery systems have been designed including suspension (5), films (6), ointments (7). These systems have drawbacks of blurred vision, no true sustained effect, may cause allergies with selective diseases, and poor patient compliance.

Hence, present research is being undertaken to make use of $\beta$ cyclodextrin complex encapsulated in vesicular system. Complexation of drug with $\beta$ - cyclodextrin will improve the solubility of azithromycin by forming complex with the drug (8). Drug delivered through the vesicles on the other hand will prolong drug delivery and provide SR/CR at the desired corneal region (9), by minimizing the systemic drainage and 
improvising the residence time. Thus, resulting in improved ocular bioavailability (10). The advantage of vesicular system does not only reside in providing prolonged effect at corneal region but also to prevent enzymatic drug metabolism at the site of action. Thus, they are examined as drug delivery carriers that can modify extent and rate of drug absorption, are chemically stable with an ability to improvise the drug performance (11). Selection of in situ gel is beneficial as it is based on the theory of phase transition in response to the environmental changes like $\mathrm{pH}$ triggered, temperature induced and ion induced systems. This system has the advantage of ease of administration as it can be instilled in sol form which converts to gel in response to the physiological condition encountered at the site of application. Thus, an enhanced retention can be assured with such delivery system because of gel formulation (12). Thus, the research is aimed to make use of vesicular in situ gel for delivery of azithromycin via ocular route that will exert dual approach of vesicles and in situ gel. Niosomes control the drug release, modify its distribution pattern as well as targeted the drug delivery to the desired site. They are expected to improvise local drug levels at desired site. Effective contact time improvises drug's bioavailability. Ocular drug delivery via niosomes modifies the rate and extent of absorption, which consequently results in reduced intraocular pressure. Thereby, providing controlled delivery. In situ gelling systems have main benefits of transporting reproducible and optimum amount, easy administration and promoting retention at precorneal site. Hence, $\beta$ - cyclodextrinniosomal in situ gel will improvise ocular bioavailability by enhancing the precorneal residence time.

\section{Materials and methods \\ Materials}

Azithromycin was gifted from Akums Pvt. Ltd., Haridwar, India as a free sample. Poloxamer 407 (Pluronic F127) was obtained from BASF Corporation, New Jersy, USA. $\beta$ cyclodextrin, sodium dihydrogen phosphate, cholesterol, span 60 were procured from S.D. Fine Chem. Limited, Mumbai, India. Cetyl pyridinium chloride monohydrate and carbopol 934P were purchased from Central Drug House, New Delhi, India. Tetrahydrofuran (THF), sodium chloride, methanol, and sodium hydroxide were obtained from Qualigens Fine Chem. Pvt. Ltd., Mumbai, India. Himedia Dialysis membrane 150 was procured from HiMedia Laboratories, Mumbai, India.

\section{Methods}

Preparation of complex

Kneading method

Drug and $\beta$-cyclodextrin were used in the molar ratios of 1:1 in order to form a complex. For this, $\beta$-cyclodextrin was triturated with mortar and pestle using small quantities of water to prepare a homogeneous paste. Drug was allowed to add in this and the mixture was grounded for $6 \mathrm{~h}$. The paste obtained was a dried complex, which was then pulverized and sieved (using sieve no. $60)$.

\section{Evaluation of complex}

Scanning electron microscopy (SEM)

Scanning electron micrographs of both azithromycin (ATZ) and ATZ- $\beta-C D$ complex were taken using instrument scanning electron microscope (JEOL 5400, Jeol, Tokyo, Japan). Particles coated with thin gold layer under an argon atmosphere by sputter coater unit (VG Microtech, West Sussex, UK) were examined. The coating time was 5-6 min. Surface morphology was studied at an acceleration voltage of $4 \mathrm{kV}$.

\section{Differential scanning calorimetry (DSC)}

Differential scanning calorimetry was conducted for both ATZ and ATZ- $\beta-C D$ complex using differential scanning calorimeter DSC60 controlled by TA-60 WS software (Shimadzu, Kyoto, Japan, 0-300 ${ }^{\circ} \mathrm{C}$. For measurements, $5 \mathrm{mg}$ samples were taken and study was performed at a heating rate of $10{ }^{\circ} \mathrm{C} / \mathrm{min}$ over $0-300{ }^{\circ} \mathrm{C}$ range. An inert atmosphere was created uisng nitrogen at a flow rate of $10 \mathrm{~mL} / \mathrm{min}$.

\section{Preliminary studies \\ Optimization of niosomes}

Preliminary studies were conducted on blank niosomes to optimize the method of preparation, type of surfactant and ratio of cholesterol and surfactant. To prepare niosomes, three methods were used (ether injection method, thin film hydration method and hand shaking method) in order to optimize an appropriate method. For the selection of optimum type of nonionic surfactant, niosomes were developed by adding surfactants like Span 60, Span 80 and Span 20. Further, optimum ratio of cholesterol and surfactant was selected among several ratios $(1: 2,2: 3,7: 8,8: 7)$. Optimization was done on the basis of photomicrographs taken using photomicrograph (HICON, New Delhi, India) at 40x.

\section{Equilibrium solubility}

Excess drug added to distilled water $(0.05 \% \mathrm{w} / \mathrm{v} ; 20 \mathrm{~mL})$ in a conical flask $\left(37^{\circ} \mathrm{C} \pm 0.5^{\circ} \mathrm{C}\right)$ stirred for a period of $72 \mathrm{~h}$ in a water bath shaker $(\mathrm{HICON} 囚$, New Delhi, India). Additionally, in several flasks, excess amount of drug mixed with $\beta$ cyclodextrin, cholesterol and span 60 was taken. Samples were filtered (nylon filter disc $(0.22 \mu)$ ), diluted and analyzed spectrophotometrically at $196 \mathrm{~nm}$. 


\section{Phase solubility}

Phase solubility analysis was conducted at $25{ }^{\circ} \mathrm{C}$ as per the methodology depicted byHiguchi and Connors (13). Excess drug amount added to distilled water containing $\beta$-cyclodextrin was shaken in water bath shaker at $100 \mathrm{rpm}(0.559 \times \mathrm{g})$ for $72 \mathrm{~h}$. Samples were filtered and analyzed against the blank prepared by using different concentrations of $\beta$-cyclodextrin in distilled water spectrophotometrically at $196 \mathrm{~nm}$. Stability constants were determined. Complexation efficiency (C.E) and apparent stability constant $(\mathrm{Ks})$ were determined from the slope of the phase solubility diagram as per the following equations:

$\mathrm{K}=$ Slope / So (1-Slope)

(Eq.1)

Complexation efficiency $(\mathrm{CE})=$ Slope/ $(1-$ Slope $)$

(Eq.2)

Where, So: intrinsic drug solubility

\section{Preparation of composite niosomes}

Composite niosomes of azithromycin were prepared based on $3^{2}$ full-factorial design as per Table 1. In total nine formulations were formulated using ether injection method. Surfactant and cholesterol dissolved in ether were mixed with methanol in a ratio of 4:1. To this ATZ- $\beta-C D$ complex was then added. The mixture was then injected with the help of 14-gauge needle in phosphate buffer solution ( $\mathrm{pH} 7.4)$ at $60-65{ }^{\circ} \mathrm{C}$ and stirred continuously. Ether and methanol evaporated at this temperature, leaving behind suspension of niosomes.

\section{Purification of composite niosomes}

Composite niosomes were purified using dialysis membrane technique to remove the free drug. Hi-media dialysis membrane 150 (MWCO 12-14 kDa) kept in saline solution for $2 \mathrm{~h}$ was used. Drug-loaded vesicles were placed in a dialysis bag dipped in $200 \mathrm{~mL}$ of phosphate buffer $\mathrm{pH} 6.8$ and undergo stirring using magnetic stirrer at $500 \mathrm{rpm}(13.98 \times \mathrm{g})$. Five $\mathrm{mL}$ of sample was withdrawn under sink conditions and analyzed spectrophotometrically. Purification time was optimized using statistical paired-t test at $5 \%$ level of significance.

\section{Characterization of composite niosomes}

Vesicle size, zeta potential and polydispersity index

All the prepared niosomal formulations were subjected to evaluation for vesicle size, zeta potential and polydispersity index determined by dynamic light scattering based on laser diffraction using Malvern Zetasizer (Malvern Co., UK) at $25^{\circ} \mathrm{C}$. Samples were diluted using deionized water and filtered through millipore $0.45 \mu \mathrm{m}$ pore size membrane (Milex®, Millipore Co., USA).

\section{Entrapment efficiency}

Centrifuged purified formulation using centrifuge (R-4 C, Remi centrifuge, Vasai, India, $1 \mathrm{~h}$ at 4,000 rpm $(894.0 \times \mathrm{g}))$ was used to evaluate entrapment efficiency. The sediment formed was then lysed by methanol. After filtration, the sample was assayed to determine the entrapment efficiency by following Eq. 3.

$\%$ Entrapment efficiency $=(\mathrm{Wa}-(\mathrm{Ws}+\mathrm{Wp}) / \mathrm{Wa})(\mathrm{Eq} .3)$

Where,

$\mathrm{Wa}=$ amount of drug added in the system;

Ws= amount of drug in the supernatant after centrifugation;

$\mathrm{Wp}=$ amount of drug in sediment.

\section{In vitro drug release}

Dialysis method was used to carry out in vitro drug release analysis. Niosomal suspension placed in a dialysis bag, kept in $200 \mathrm{~mL}$ phosphate buffer, $\mathrm{pH} 7.4$ was stirred at $100 \mathrm{rpm}$. Withdrawn samples were analyzed spectrophotometrically at $215 \mathrm{~nm}$ under sink conditions.

\section{Validation of experimental design}

Polynomial equation was generated using Design Expert Software version 9.0.6.2 (Stat-Ease, Minneapolis, USA Inc.) for dependent variable. Extra design check point formulations were developed to validate the model and characterized for percent entrapment efficiency. The findings were then comparedwith

Table $13^{2}$ Factorial design for the development of ATZ- $\beta$-CD complex encapsulated composite niosomes

\begin{tabular}{ccccc}
\hline $\begin{array}{c}\text { Formulati } \\
\text { on code }\end{array}$ & $\begin{array}{c}\text { Cetylpyridinium } \\
\text { chloride }(\% \mathbf{w} / \mathbf{v})\end{array}$ & $\begin{array}{c}\text { Cholesterol } \\
(\% \mathbf{w} / \mathbf{v})\end{array}$ & $\begin{array}{c}\text { Span 60 } \\
(\% \mathbf{w} / \mathbf{v})\end{array}$ & $\begin{array}{c}\text { Di-ethyl ether: } \\
\text { methanol }\end{array}$ \\
\hline NF1 & 0.1 & 0.1 & 0.1 & $4: 1$ \\
\hline NF2 & 0.1 & 0.1 & 0.5 & $4: 1$ \\
\hline NF3 & 0.1 & 0.1 & 1 & $4: 1$ \\
\hline NF4 & 0.1 & 0.5 & 0.1 & $4: 1$ \\
\hline NF5 & 0.1 & 0.5 & 0.5 & $4: 1$ \\
\hline NF6 & 0.1 & 0.5 & 1 & $4: 1$ \\
\hline NF7 & 0.1 & 1 & 0.1 & $4: 1$ \\
\hline NF8 & 0.1 & 1 & 0.5 & $4: 1$ \\
\hline NF9 & 0.1 & 1 & 1 & $4: 1$ \\
\hline
\end{tabular}


the predicted value and evaluated statistically using pooled t- test at $5 \%$ level of significance.

\section{Selection of optimized formulation}

Optimized formulation was selected based on highest desirability value and loaded into suitable gel base.

\section{Preparation of in situ gel}

Gel based formulations were prepared by cold method. Niosomal pellets were added to presoaked carbopol $934 \mathrm{P}$ and stirred at room temperature $\left(25^{\circ} \mathrm{C}\right)$. The dispersion was then cooled at $4{ }^{\circ} \mathrm{C}$ by keepig in a refrigerator. Polaxamer 407 was added slowly as per the composition (Table 2) to the above dispersion under continuous stirring (thermostatically controlled magnetic stirrer (S.M. Scientific instruments Pvt Ltd., New Delhi, India)), maintaining the temperature at $4{ }^{\circ} \mathrm{C}$. Suspensions were then stored in a refrigerator overnight to get clear solutions. Thereafter, formulations were stored in a refrigerator so that they remain in sol form.

\section{Characterization of in situ gel}

\section{Clarity, $\mathrm{pH}$ and percent drug content}

Clarity of the developed gel based formulations was determined before and after gelation by visual examination of the formulations under light alternatively against white and black background. $\mathrm{pH}$ of the developed gel formulations was determined using Digital $\mathrm{pH}$ meter model 111 E (HICON®, New Delhi, India). For drug content, weighed amount (50 mg) of ATZ loaded ocular niosomal in situ gel formulations were diluted using phosphate buffer, $\mathrm{pH}, 7.4$. The resultant dispersion was vortexed using Vortex shaker (HICON®, New Delhi, India) and shaken for $10 \mathrm{~min}$. The volume was made up to $5 \mathrm{~mL}$ with phosphate buffer $\mathrm{pH}, 7.4$ and analyzed spectrophotometrically at $215 \mathrm{~nm}$. All readings were taken in triplicate.

Table 2 Composition of azithromycin (ATZ) loaded composite niosomal in situgels

\begin{tabular}{cccc}
\hline $\begin{array}{c}\text { Formulation } \\
\text { Code }\end{array}$ & $\begin{array}{c}\text { Polaxamer } \\
\mathbf{4 0 7} \\
(\boldsymbol{\%} \mathbf{w} / \mathbf{v})\end{array}$ & $\begin{array}{c}\text { Carbopol } \\
\mathbf{9 3 4 P} \\
(\boldsymbol{\%} \mathbf{w} / \mathbf{v})\end{array}$ & $\begin{array}{c}\text { ATZ loaded } \\
\text { composite } \\
\text { niosomes (\% w/v) }\end{array}$ \\
\hline NG1 & 12 & 2 & 0.5 \\
\hline NG2 & 14 & 2 & 0.5 \\
\hline NG3 & 16 & 2 & 0.5 \\
\hline NG4 & 18 & 2 & 0.5 \\
\hline NG5 & 20 & 2 & 0.5 \\
\hline
\end{tabular}

\section{In vitro gelation}

In vitro gelling efficiency was determined using a transparent vial containing $10 \mathrm{~mL}$ of niosomal gelling solution and a magnetic bar was placed in a low-temperature water bath (HICON®, New Delhi, India). A thermometer with an accuracy rating of $0.1{ }^{\circ} \mathrm{C}$ was immersed in the niosomal gelling solution. The solution was heated at the rate of $1{ }^{\circ} \mathrm{C} / 1-2 \mathrm{~min}$ with constant stirring of $5 \mathrm{rpm}$. The temperature at which the magnetic bar completely stopped movement because of gelation was regarded as the phase transition temperature. Each study was done in triplicate. Gelling time is the time for first detection of gelation was also recorded.

\section{Viscosity}

The rheological characteristics of in situ gelling system was evaluated by Brookfield viscometer DV- II+ pro (Brookfield Engineering Laboratories, Inc, MA) coupled with T spindle S96 at physiological condition i.e. $37 \pm 2{ }^{\circ} \mathrm{C}$.

\section{Ex vivo permeability}

Ex vivo permeability study of all ATZ- $\beta-C D$ complex loaded niosomal gel (NG1-NG5) was carried out using fabricated Franz diffusion cell consisting of donor and receptor compartment separated by dialysis membrane of pore size 0.22 $\mu \mathrm{m}$ (Himedia Ltd., Mumbai, India). Gel based formulations equivalent to $1 \% \mathrm{w} / \mathrm{v}$ of ATZ - $\beta$-CD complex loaded niosomes placed in donor compartment and receptor compartment was filled with phosphate buffer, $\mathrm{pH} 7.4$ and constantly stirred using magnetic stirrer. Temperature was kept constant at $37 \pm 1.0{ }^{\circ} \mathrm{C}$ in order to mimic body conditions. One $\mathrm{mL}$ sample was withdrawn through the sampling port of the diffusion cell at 1,2, $3,4,5,6,7$ and $8 \mathrm{~h}$ time intervals and analyzed spectrophotometrically at $215 \mathrm{~nm}$. An equal volume of fresh phosphate buffer, $\mathrm{pH} 7.4$ was added into the receptor compartment after each sampling to maintain the sink conditions.

\section{Selection and characterization of optimized gel formulation}

Optimized gel formulation was selected on the basis of formulation that showed clarity, maximum gelling capacity along with optimum viscosity, maximum drug content and minimum ex- vivo permeation. Optimized formulation was then characterized for in vitro mucoadhesion, and histological analysis.

\section{In vitro mucoadhesion}

Mucoadhesive strength was determined as per the method given by Qi et al., (14). The sclera was obtained from goat eye collected from local butcher shop and washed with normal buffer saline and kept in freshly prepared Ringer's salt solution $\left(37^{\circ} \mathrm{C}, 15 \mathrm{~min}\right)$. Excised scleral tissue was attached to the under surface of pan of balance and equilibrated. Optimized in situ gel formulation was placed in sample holder placed on water bath at 
$37^{\circ} \mathrm{C}$. $\mathrm{pH}$ of sample was adjusted to 7.4. Equilibrium of balance was disturbed by putting a weight of $5.0 \mathrm{gm}$ on the left pan of balance to assure complete contact between gel surface and scleral tissue. The weight was then removed and water was added drop wise to the beaker placed on right pan with the help of burette. Water was added continuously until the gel and corneal tissue got detached. The mucoadhesive force was determined by calculating detachment stress (dynes $/ \mathrm{cm}^{2}$ ), which gives the value of minimal force required to detach cornea from the gel.

Detachment stress $=\mathrm{m} \times \frac{\mathrm{g}}{\mathrm{A}}$

(Eq.4)

Where,

$\mathrm{m}=$ the weight added to balance in gram.

$\mathrm{g}=$ acceleration due to gravity taken as $980 \mathrm{~cm} / \mathrm{sec}^{2}$

$\mathrm{A}=$ area of exposed tissue.

\section{Comparative in vitro release analysis}

In vitro release study of optimized niosomes loaded in situ gel, niosomes, and azithromycin eye drop (Zithromax; Allembic Pharmaceuticals, Vadodara, India) was performed using dialysis bag method. Sample were placed in dialysis bag and kept in a $200 \mathrm{~mL}$ of phosphate buffer $\mathrm{pH}, 7.4$, stirred at $100 \mathrm{rpm}$ using magnetic stirrer $\left(37 \pm 2{ }^{\circ} \mathrm{C}\right)$. Samples were drawn and analyzed spectrophotometrically under sink conditions.

\section{Histological analysis}

Histological analysis was performed to evaluate the effect of formulations on scleral integrity. Collected whole eye balls of goat were transported immediately to the laboratory in normal buffered saline in cold condition within $1 \mathrm{~h}$ of slaughtering. Rinsed sclera from excised eyes was incubated for $30 \mathrm{~min}$ in optimized NG5 formulation at $34{ }^{\circ} \mathrm{C}$, phosphate buffer saline (PBS), pH 7.4 as negative control and 75\% isopropyl alcohol in PBS as positive control was taken as reference. The sclera was washed with PBS, pH 7.4 and immediately fixed with $10 \% \mathrm{v} / \mathrm{v}$ formalin solution for $24 \mathrm{~h}$. The sclera was dehydrated with ethyl alcohol and xylene, put in melted paraffin, and solidified in block forms. Cross sections (< 1mm) were observed under microscopy for any histological changes after staining with hematoxylin and eosin (15).

\section{Results}

Preparation and evaluation of complex

Drug- $\beta$-cyclodextrin complex was prepared by kneading method and undergo characterization for surface morphology and DSC.

\section{Scanning electron microscopy}

Scanning electron microscopy is thought to be a mode to study the morphological changes at surface. Scanning electron microphotographs of azithromycin and ATZ- $\beta$-CD complex are depicted in Figure 1. ATZ was appeared as regular three dimensional crystals (Figure 1A). A major morphological change has been observed in the complex formation. However, the original morphological diagnosis of the materials diminished and was unable to undergo differentiation. Crystalline structure of drug has perished (Figure 1B) and the amorphous agglomerate is formed after complex formation. These modifications in shape of the particles in binary complexes indicated the presence of new solid phase.

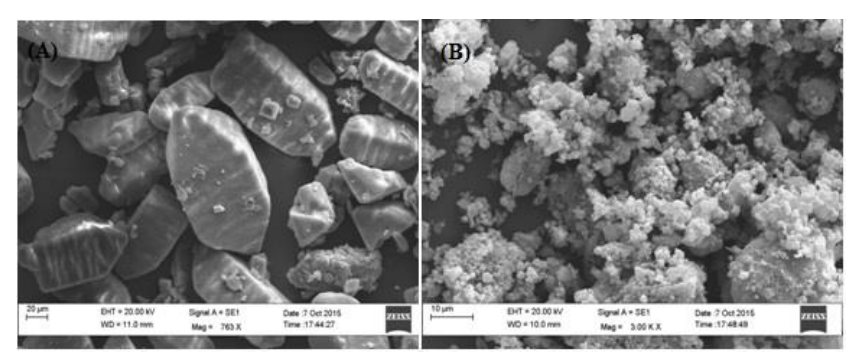

Figure 1 SEMphotomicrograph of (A) Azithromycin (ATZ), (B) ATZ- $\beta$-CD complex

\section{Differential scanning calorimetry}

DSC was conducted to trace any form of drug- excipient interaction. Figure 2A depicted sharp endothermic peak at $110.54{ }^{\circ} \mathrm{C}$ (due to ATZ) because of the melting of drug whereas broad endothermic peak at $272.79{ }^{\circ} \mathrm{C}$ showed thermal decomposition of drug. In case of complex formation (ATZ- $\beta$ CD) (Figure 2B), peak of drug was not observed at $110.54^{\circ} \mathrm{C}$. Thus, suggesting the fact that ATZ was dispersed molecularly in complex and exothermic peak at $353.53^{\circ} \mathrm{C}$ had proved the thermal decomposition of complex form.

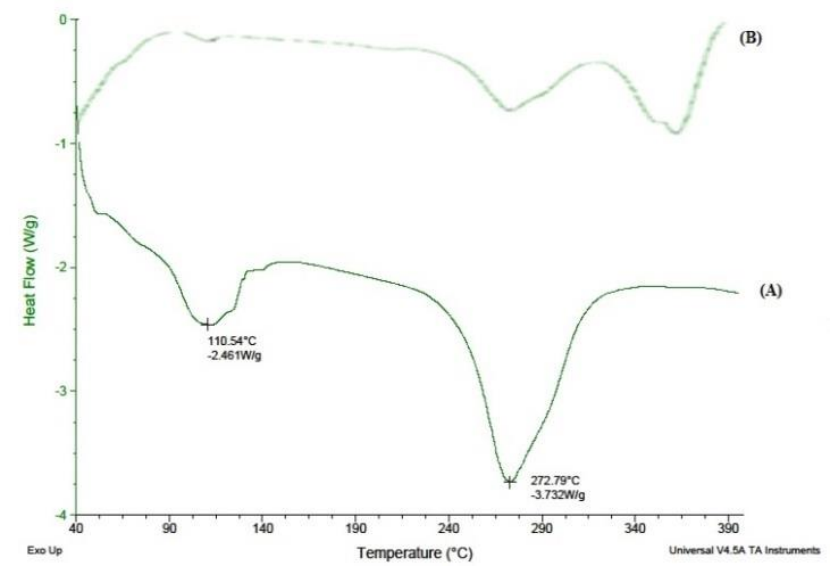

Figure 2 DSC thermogram of (A) Azithromycin (ATZ), (B) ATZ- $\beta$-CD 
Preliminary optimization studies on blank composite niosomes Optimization of niosomes

Blank niosomes were visualized and the photomicrographs revealed that thin film hydration method vesicles were irregular in size/shape and possessed undefined structures while vesicles formation could not be facilitated by hand shaking method (Figure 3 (I) A). Niosomes prepared by ether injection method (Figure 3(I) C) were spherical in shape with structure uniformity.

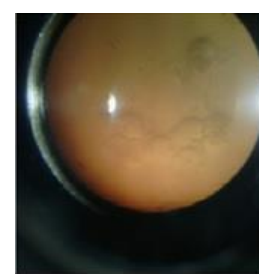

[I] (A)

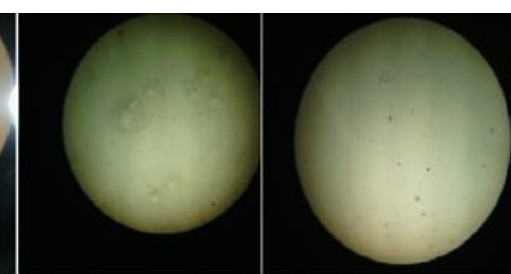

(B)

(C)

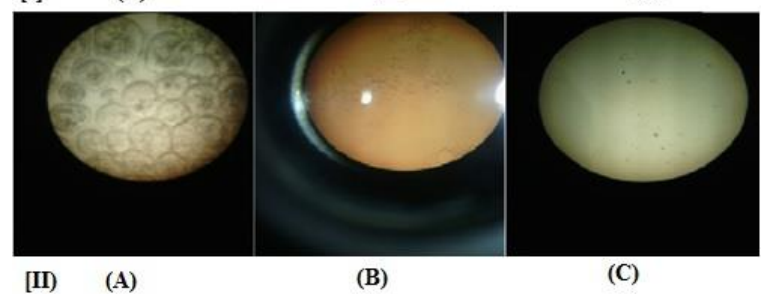

[II) (A)

(B)

(C)

Figure 3 Photomicrographs of blank niosomes prepared by [I] (A) Hand shaking method, (B) Thin film hydration method, (C) Ether injection method; [II] (A) Span 20; (B) Span 80; (C) Span 60

Thus, ether injection method was optimized for preparing niosomes. Next, to select appropriate surfactant among several added surfactants, span 60 was selected (Figure 3(II) C). As, it was observed that niosomes prepared using spam 60 were uniform in shape with rigidity and firmness in their structure (Figure 3(II) C). Whereas vesicles did not form in case of span 20 (Figure 3(II) A) and in presence of span 80 vesicles were non-uniform size and shape (Figure 3(II) B). Thus, span 60 was selected as an optimum surfactant. Further, appropriate ratio of span 60 and cholesterol was selected by changing the ratio at $1: 1,3: 2,7: 8$ and 8:7. Out of these ratios $1: 1$ selected optimum for preparation of niosomes because most of the vesicles were uniform in shape and of similar size in comparison with other ratios prepared niosomes. Thus, optimum ratio of span 60 and cholesterol was identified to be $1: 1$.

\section{Equilibrium solubility}

Solubility of drug in distilled water was determined as 0.0851 $\mathrm{mg} / \mathrm{ml}$. Addition of $\beta$-cyclodextrin and span 60, depicted the percent solubility enhancement as 75.30 and 42.32, respectively (Table 3 ). $\beta$-cyclodextrin improves the solubility of azithromycin by forming soluble complexes with drug by enclosing the drug in its hydrophobic cavity (16)whereas span
60 increased the solubility due to its surfactant nature.Hydrophilic head and hydrophobic tail in its structure decrease the surface tension in aqueous media and facilitate solubilization.Methanol as a solvent depicted a solubility enhancement due to their cosolvent action. Thus, it may be put into conclusion that the excipients used in formulating composite niosomes, represents solubility enhancement and hence higher drug entrapment. On the basis of these findings, composite niosomes were developed.

\section{Phase solubility}

Phase solubility study was performed to optimize the levels of $\beta$-cyclodextrin for experimental design as well to find stoichiometric ratio of $\beta$-cyclodextrin and azithromycin in order to prepare binary complex to explore its utility as a carrier. Azithromycin in presence of $\beta$-cyclodextrin indicated increased drug solubility on increasing the concentration of $\beta$ cyclodextrin. Phase solubility profile was plotted using apparent equilibrium concentration or solubility of drug against increasing concentration of $\beta$-cyclodextrin (Figure 4). Apparent drug solubility enhanced linearly with $\beta$-cyclodextrin over the entire concentration range. Linearity was considered as the characteristic of AL- type system that suggested the formation of a complex (water soluble).

Table 3 Solubility data of azithromycin in distilled water in the presence of excipients

\begin{tabular}{cccc}
\hline $\begin{array}{c}\text { Sample } \\
\text { composition }\end{array}$ & $\begin{array}{c}\text { Strength of } \\
\text { excipients } \\
(\% \mathbf{\%} / \mathbf{v})\end{array}$ & $\begin{array}{c}\text { Solubility } \\
(\mathbf{m g} / \mathbf{m l})\end{array}$ & $\begin{array}{c}\text { \% solubility } \\
\text { Enhancement }\end{array}$ \\
\hline $\mathrm{D}$ & 0.05 & 0.0851 & - \\
\hline $\mathrm{D}+\mathrm{SP}-60$ & $0.05+0.5$ & 0.147 & $42.32 \pm 1.08$ \\
\hline $\mathrm{D}+\mathrm{CHO}$ & $0.05+0.5$ & 0.104 & $18.33 \pm 0.98$ \\
\hline $\mathrm{D}+\mathrm{MeOH}$ & $0.05+20$ & 0.529 & $83.91 \pm 1.15$ \\
\hline $\mathrm{D}+\beta-\mathrm{CD}$ & $0.05+0.5$ & 0.346 & $75.30 \pm 1.34$ \\
\hline $\mathrm{D}+\mathrm{SP}-$ & $0.05+0.5+0.5$ & 1.077 & $92.10 \pm 1.01$ \\
$60+\mathrm{CHO}+$ & $+20+0.5$ & & \\
$\mathrm{MeOH}+\beta-$ & & & \\
$\mathrm{CD}$ & & & \\
\hline
\end{tabular}

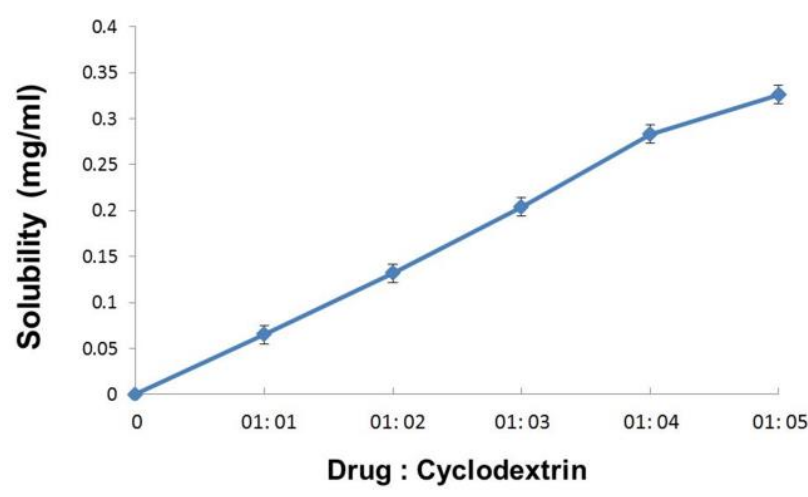

Figure 4 Phase solubility diagram with varying ratio of drug and cyclodextrin 
Further, 0.896 obtained as a slope value of the complex, indicated the formation of inclusion complex (1:1). K1:1 was observed as $168 \mathrm{M}^{-1}$. The value of inclusion complex suggested that the complex formed was very less stable which may increase the processof dissociation of drug. Phase solubility curve did notconfirm the formation of complex.These curves however, depicted that the increasing concentration of solubilizer influence the drug solubility (17). Based on these results, $\beta$-cyclodextrin-azithromycin ratio was selected as 1:1 for designing composite niosomes.

\section{Preparation and evaluation of niosomes}

Niosomes were developed by ether injection method as described earlier according to the formulation design as given in table 1and evaluated.

\section{Vesicles Size, Zeta Potential and Polydispersity index (PDI)} Vesicle size of ATZ- $\beta-C D$ loaded niosomes varied from $306 \pm$ 3.05 to $2212 \pm 4.35 \mathrm{~nm}$ with PDI value ranging from $0.115 \pm$ $5.51-0.677 \pm 0.02$. Di cetyl pyridinium chloride containing NFs (NF1-NF9) showed positive zeta potential values of 24.2 \pm 0.23 to $45.3 \pm 0.25 \mathrm{mV}$ due to hydrophilic cationic nature of it.

\section{Entrapment efficiency and in vitro drug release}

Percent entrapment efficiency was observed to be $30.07 \pm 5.09$ $-78.17 \pm 1.81$ as shown in Table 4.In vitro release study was conducted to correlate the functioning of a system with its in vivo effectiveness. In vitro drug release (Figure 5) was found to be varied from $23.95 \pm 2.30$ to $76.28 \pm 2.22 \%$. Higher drug release was observed in NF1 formulation $(76.28 \pm 2.22 \%)$.

\section{Validation of Experimental Design}

Design Expert Software version 9.0.6.2 (Stat-Ease, Minneapolis, USA Inc.) was used to validate $3^{2}$ factorial design. The developed model was described using polynomial equation. This can be given as follows:

$\%$ Entrapment efficiency $=+84.18+4.78 x_{1}-2.10 x_{2}$ (Eq.5)

The presence of positive sign indicated the positive effect of cholesterol $\left(\mathrm{x}_{1}\right)$ on percent entrapment efficiency.Negative sign in numerical values showed the inhibitory effect of span 60 $\left(\mathrm{x}_{2}\right)$ on the response. Extra design check point formulation (NF10) showed percent entrapment efficiency to be $76.59 \pm$ $1.06 \%$.

\section{Selection of optimized formulation}

Formulation NF2 showed maximum desirability (equal to 0.807). Also, exhibit optimum percent cumulative drug release $(73.09 \pm 2.11 \%)$, entrapment efficiency $(78.17 \pm 1.81 \%)$ with optimum particle size $(306 \pm 3.05 \mathrm{~nm})$ and optimum PDI value $0.218 \pm 5.51$. Thus, NF2 was considered as best among all the formulations. Optimized formulation was further incorporated into gel and characterization.

\section{Preparation and characterization of in situ gel}

Optimized ATZ loaded niosomal formulation NF2 was incorporated into temperature controlled in situ gels prepared by cold method. Gels were prepared with the aim to achieve more controlled release of niosomes from gelling system and increase ocular residence time.

Table 4 Pharmacotechnical characterization of ATZ- $\beta$-CD loaded composite niosomes (NF1-NF9)

\begin{tabular}{cccccccc}
\hline $\begin{array}{c}\text { Formulat } \\
\text { ion code }\end{array}$ & $\begin{array}{c}\text { Vesicle size } \\
(\mathbf{n m})\end{array}$ & $\begin{array}{c}\text { Zeta potential } \\
(\mathbf{m V})\end{array}$ & $\begin{array}{c}\text { Polydispers } \\
\text { ity index }\end{array}$ & $\begin{array}{c}\text { \% Entrapment } \\
\text { efficiency }\end{array}$ & \% CDR & $\begin{array}{c}\text { Kinetic } \\
\text { model }\end{array}$ & $\mathbf{r}^{2}$ \\
\hline $\mathrm{NF} 1$ & $1258 \pm 7.21$ & $38.5 \pm 0.43$ & $0.115 \pm 5.51$ & $75.51 \pm 2.40$ & $76.28 \pm 2.22$ & Higuchi & 0.9972 \\
\hline $\mathrm{NF} 2$ & $306 \pm 3.05$ & $45.3 \pm 0.25$ & $0.218 \pm 5.51$ & $78.17 \pm 1.81$ & $73.09 \pm 2.1$ & Higuchi & 0.9948 \\
\hline $\mathrm{NF3}$ & $714 \pm 3.60$ & $32.6 \pm 0.20$ & $0.279 \pm 3.79$ & $74.36 \pm 0.07$ & $54.82 \pm 1.79$ & Higuchi & 0.9877 \\
\hline $\mathrm{NF} 4$ & $2212 \pm 4.35$ & $30.0 \pm 0.20$ & $0.555 \pm 0.02$ & $68.84 \pm 0.52$ & $43.47 \pm 0.91$ & Higuchi & 0.9875 \\
\hline $\mathrm{NF5}$ & $877 \pm 4.16$ & $41.4 \pm 0.23$ & $0.647 \pm 0.03$ & $65.80 \pm 0.25$ & $53.64 \pm 1.33$ & $\begin{array}{c}\text { Korsmeyer } \\
\text { and Peppas }\end{array}$ & 0.9986 \\
\hline $\mathrm{NF6}$ & $553 \pm 3.51$ & $35.4 \pm 0.20$ & $0.677 \pm 0.02$ & $63.65 \pm 0.69$ & $34.91 \pm 1.55$ & Higuchi & 0.9610 \\
\hline $\mathrm{NF} 7$ & $876 \pm 4.35$ & $32.7 \pm 0.057$ & $0.577 \pm 0.02$ & $59.23 \pm 1.14$ & $36.10 \pm 6.8$ & Higuchi & 0.9759 \\
\hline $\mathrm{NF} 8$ & $847 \pm 2.51$ & $28.4 \pm 0.35$ & $0.653 \pm 0.04$ & $41.28 \pm 4.12$ & $23.95 \pm 2.30$ & $\begin{array}{c}\text { Korsmeyer } \\
\text { and Peppas }\end{array}$ & 0.9934 \\
\hline $\mathrm{NF9}$ & $2041 \pm 3.51$ & $24.2 \pm 0.23$ & $0.662 \pm 0.04$ & $30.07 \pm 5.09$ & $27.55 \pm 1.26$ & $\begin{array}{c}\text { Korsmeyer } \\
\text { and Peppas }\end{array}$ & 0.9944 \\
\hline NF10* & $634 \pm 3.60$ & $46.9 \pm 0.81$ & $0.566 \pm 0.01$ & $76.59 \pm 1.06$ & $61.065 \pm 2.67$ & First & 0.9884 \\
\hline NF10* Extra design check point formulation. & & & & &
\end{tabular}




\section{Clarity, $\mathrm{pH}$ and drug content}

All the formulations were found to be clear, there was no residue matter present which can harm sclera tissue and affect the syringeability of the formulations. $\mathrm{pH}$ of all formulations before gelation was found to be towards acidic side $(6.30 \pm 0.03$ to 6.83 $\pm 0.03)$ and after gelation it changes $(6.28 \pm 0.01$ to $7.21 \pm 0.02)$ (Table 5). These changes confirmed the ability of conversion from sol to gel on ocular instillation (15).

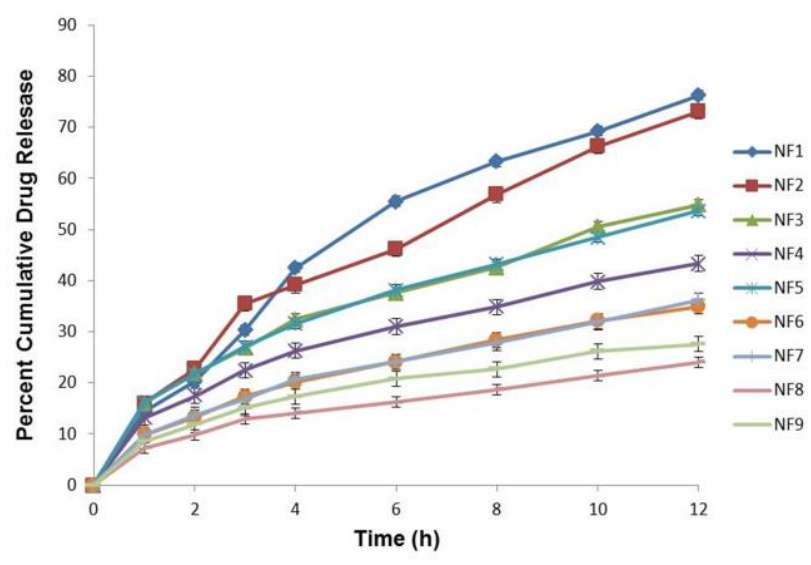

Figure 5 In vitrodrug release profiles in phosphate buffer, $\mathrm{pH} 7.4$

Further, $\mathrm{pH}$ range of 6.8 to 7.4 is thought to be acceptable and safe for ocular delivery. Drug content lies in the range from $63.44 \pm 0.68$ to $80.79 \pm 1.04 \%$.

\section{In vitro gelation and viscosity}

Gelling temperature lies in between $28.33 \pm 0.57-32.00 \pm$ $.0 .0^{\circ} \mathrm{C}$ for all the formulations (Table 5). A gelling temperature is recorded to be optimum in the range of $25{ }^{\circ} \mathrm{C}$ to $37{ }^{\circ} \mathrm{C}$. Viscosity of all the formulations lies in between $38 \times 10^{3}-45 \mathrm{x}$ $10^{3} \mathrm{cps}$.

\section{Ex-vivo permeability}

Ex vivo permeation study of in situ ocular gels was performed to evaluate the role of niosomes in improvising permeation across the sclera. \% CDP varied widely between $37.07 \pm 2.06$ to $51.34 \pm 0.89$.

\section{Selection and characterization of optimized formulation}

NG5 possessing highest drug content, maximum gelling capacity, and lowest permeation capacity was found to be best among all the formulations in order to exert localize effect in ocular delivery. Optimized formulation was then characterized for in vitro mucoadhesion and histological study.

In vitro mucoadhesion and comparative in vitro release analysis

Mucoadhesive strength of niosomal in situ gel (NG5) was found to be 3462.67 dynes $/ \mathrm{cm}^{2}$ which was approximately 23 folds greater than ocular shear force $\left(150\right.$ dynes $\left./ \mathrm{cm}^{2}\right)$. In vitro release profile of NG5 was compared with that of NF2, Zithromax ${ }^{\circledR}$ eyedrop and subjected to kinetic analysis. The role of niosomes and in situ gel in improvising the release through sclera was demonstrated. NG5 was able to sustain the drug release and achieved $65.45 \pm 0.87 \%$ in $8 \mathrm{~h}$ (Figure 6) that was comparable with CDR of NF2 that achieved $67.32 \pm 1.27$

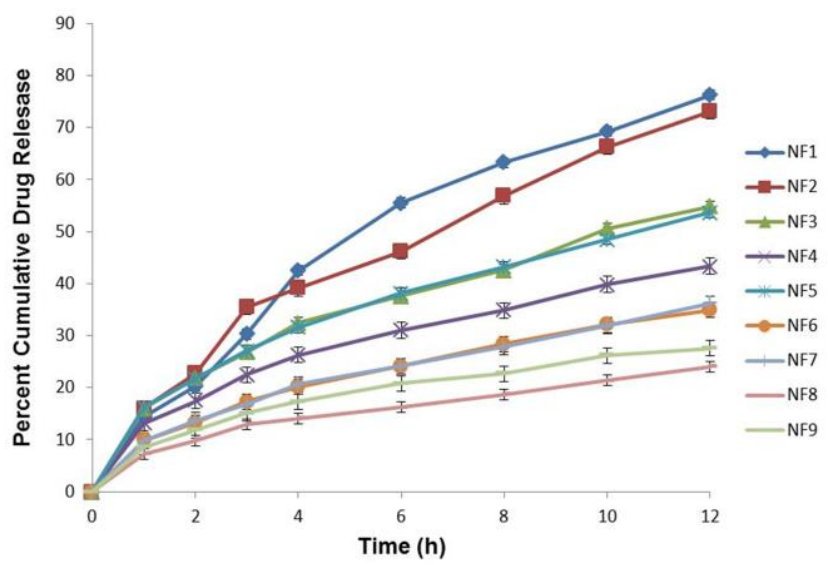

Figure 6 Comparative in vitrorelease of azithromycin from optimized niosomal formulations, Niosomalin situ gel, and marketed formulation in phosphate buffer, $\mathrm{pH} 7.4$

$\%$ in the same time period. Release of Zithromax® (marketed preparation)showed instantaneous release of drug as drug present in solution form. Thus, maximum drug released within $2 \mathrm{~h}$ and only $54 \%$ of drug was released from marketed solution. So, on comparing the release of Zithromax ${ }^{\circledR}$ with NG5, in situ gel follows zero order release. Zero order release was followed by gel as drug release undergoes retardation when gel forms and follows more constant release of drug. On the other hand, marketed preparation releases the drug in an uncontrolled order and release maximum drug within in $2 \mathrm{~h}$. In case of NF2, niosomes showed better release of drug because it follows sustained release of drug. Whereas in situ gel based NG5 formulation was found to be best among all three formulation in case of retaining and release manner of drug.

\section{Histological analysis}

Effect of optimized in situ gel formulation (NG5) on the structural features of scleral tissue was evaluated (Figure 7). After incubating scleral cross-section in 75\% isopropyl alcohol (Figure 7 (C)), narrow intercellular spaces widened with deformation. Further, superficial epithelial cells undergo distortion and got detached from the tissue assembly. Incubation of sclera with NG5 (Figure 7 (B)) did notrevealed any destructive effect on corneal epithelium, stromal structure. Thus, maintained the corneal integrity. The above results showed by optimized formulation were found to be similar to the effect shown after incubating scleral tissue in PBS, pH 7.4 (Figure7(A)). 
Table 5 Characterization of composite niosomal loaded in situgel (NG1-NG5)

\begin{tabular}{ccccccc}
\hline $\begin{array}{c}\text { Formulation } \\
\text { Code }\end{array}$ & $\begin{array}{c}\text { Temperature } \\
\text { (Celsius) }\end{array}$ & $\begin{array}{c}\text { Gellingtime } \\
\text { (seconds) }\end{array}$ & pH (sol) & pH (gel) & $\begin{array}{c}\text { Percent Drug } \\
\text { content }\end{array}$ & \% CDP \\
\hline NG1 & $28.33 \pm 0.58$ & $74 \pm 1.22$ & $6.83 \pm 0.03$ & $6.90 \pm 0.01$ & $63.44 \pm 0.69$ & $51.34 \pm 0.89$ \\
\hline NG2 & $31.66 \pm 1.07$ & $40 \pm 2.00$ & $6.30 \pm 0.03$ & $7.18 \pm 0.04$ & $65.73 \pm 0.68$ & $48.07 \pm 0.90$ \\
\hline NG3 & $32.00 \pm 0.00$ & $33 \pm 1.52$ & $6.34 \pm 0.01$ & $7.21 \pm 0.02$ & $75.16 \pm 0.80$ & $46.46 \pm 0.27$ \\
\hline NG4 & $30.66 \pm 1.15$ & $22 \pm 1.00$ & $6.40 \pm 0.01$ & $6.41 \pm 0.02$ & $76.38 \pm 2.07$ & $38.68 \pm 1.52$ \\
\hline NG5 & $32.00 \pm 0.00$ & $16 \pm 0.57$ & $6.40 \pm 0.03$ & $6.28 \pm 0.005$ & $80.796 \pm 1.04$ & $37.07 \pm 2.06$ \\
\hline
\end{tabular}
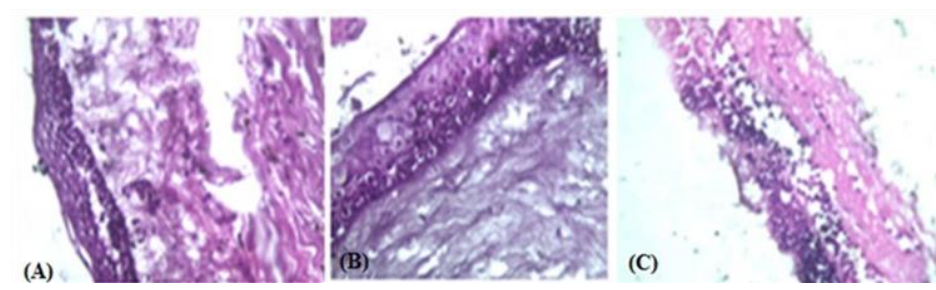

Figure 7 Histological cross section of excised goat sclera, stained with hematoxylin-eosin after incubation in (A) PBS, pH 7.4 (negative control); (B) NG5; (C) 75\% isopropyl alcohol (positive control).

\section{Discussion}

Niosomes loaded with azithromycin- $\beta$-CD complex were developed based on $3^{2}$ full factorial design using ether injection method. All the nine formulations were then evaluated for vesicles size, zeta potential, polydispersity index (PDI), percent entrapment efficiency, and percent cumulative drug release.

Formulation NF1 to NF3 containing least amount of cholesterol showed minimum particle size. This can be revealed based on the concentration of cholesterol. As amphipathic feature of cholesterol is an important parameter to understand the effect of increasing cholesterol content on average increment in particle size of niosomes. Cholesterol can be embedded into bilayer membrane by orienting its hydrophilic head towards the aqueous surface and aliphatic chain arranged parallel to the hydrocarbon chains in centre of bilayer (18). At low concentration of cholesterol, it can be expected that cholesterol get arranged surfactant monomers in close packing with increasing curvature and thus, minimizing the particle size (as observed in NF1 to NF3). However, upon increasing the cholesterol content, with its known lipophilic nature $(\log P$ 7.02), it would have enhanced the hydrophobicity of the bilayer membrane and disturbed the vesicular membrane, thus, improvised the radius to provide a more thermodynamic stable form, which is finally found in average particle size of NF4 to NF6. Similarly, in case of NF7 to NF9, increment in radius was observed but in higher amount because of the presence of higher cholesterol concentration. PDI is depicted to be the measurement of homogeneity which dictates the distribution of vesicles. A value of less than 0.3 PDI value depicts the homogenous particle distribution (19). PDI value was found to be minimum from NF1 to NF3 (0.115 \pm 5.507 to $0.279 \pm 3.785$ ) among niosomal vesicles which signifies the homogenous nature of first three formulation and higher PDI value as found in NF4 to NF9 defined the heterogeneous nature formulation which might be due to aggregation of the particle. Zeta potential values highlight the particle surface charge that defines the degree of repulsion between the adjacent particles. Its value largely affected the stability of nanoparticles through the electrostatic repulsion between the particles. Zeta potential of -30 to $+30 \mathrm{mV}$ signifies that a system is stable $(20,21)$.

The zeta potential values of ATZ- $\beta$-CD conferred physical stability to the system and positive value indicates the stability of niosomes by electrostatic repulsive forces (22). Zeta potential values also depicted that NFs maintains their charge even in the presence of drug molecules thus, suggesting that drug molecules are uniformly dispersed within the niosomes (23). Further, depiction of entrapment efficiency highlighted that niosomal formulations containing optimum concentration of cholesterol showed highest entrapment as shown in formulations (NF1-NF3). This can be attributed to the presence of cholesterol which exhibited cement leaking quality. As cholesterol concentration increased from $0.1-0.5 \% \mathrm{w} / \mathrm{v}$ (in NF4-NF6), entrapment efficiency decreased from $68.84 \pm 0.5$ to $63.65 \pm 0.69 \%$. Beyond a level of $0.5 \% \mathrm{w} / \mathrm{v}$, disruption of regular bilayer structure occurred that led to drug loss (24). As observed in concentration of 0.5 to $1 \% \mathrm{w} / \mathrm{v}$ in (NF7- NF9), drug entrapment efficiency decreased from $59.23 \pm 1.14$ to $30.07 \pm 5.09 \%$. Thus, with increased cholesterol concentration, entrapment efficiency decreased because of cholesterol intercalation in bilayers. Also, higher amount of cholesterol, for packing space within the bilayer may compete with the drug. As $0.1 \%$ w/v of cholesterol present in NF1-NF3, these formulations showed better entrapment efficiency in 
comparison with NF4 to NF6 because less drug entrapped in niosomes as drug compete with cholesterol for entrapment in bilayers. Similarly, least drug entrapped in NF7-NF9 because of highest concentration of cholesterol in all these formulations. So, upon increasing the concentration of cholesterol, stability and bilayer hydrophobicity increased and permeability got reduced. This may also led to an efficient hydrophobic drug trapping into bilayers (25). The findings of in vitro drug release demonstrated that upon increasing the cholesterol concentration, entrapment efficiency decreased, thus, percent drug release also decreased. As drug release dependent on the entrapment efficiency (24).

Formulations containing least amount of cholesterol showed higher percent of drug release because of higher drug entrapment. Reduced concentration of cholesterol resulted in the reduced membrane permeability that resulted in lesser drug elution via vesicles (26). Data of in vitro release was then subjected to kinetic analysis to understand the mechanism of release. In vitro drug release via niosomes was found to follow Higuchi model kinetics. After evaluating all the parameters, experimental design was then subjected to validation using Design Expert Software version 9.0.6.2 (Stat-Ease, Minneapolis, USA Inc.). Transformed polynomial equation indicated no significant difference $(\mathrm{p}>0.05)$ between the two values (experimental and predicted value $(79.40 \%)$ ) upon evaluation using pooled t- test. Thus, it was concluded that design has been validated. Thus, based on maximum desirability (equal to 0.807 ), formulation NF2 was selected as an optimized formulation and developed in the form of in situ gel. Developed gels were then evaluated for clarity, $\mathrm{pH}$, drug content, in vitro gelation

For the development of ocular in situ gel, clarity, $\mathrm{pH}$ and gelling capacity become the important prerequisite parameters. Thus, the formed gel should be clear and should not cause blurring of vision. As for a successful in situ gelling system, $\mathrm{pH}$ of the solution should be such that it allows instillation of liquid solution easily. This further will transform from sol to gel on coming in contact with ocular physiological conditions and could not cause any irritation in ocular area (25). However, at lower value of gelling temperature (less than $25^{\circ} \mathrm{C}$ ), gel formation occurs at room temperature. This might led to several problems in handling, manufacturing, and administration. At higher values of more than $37^{\circ} \mathrm{C}$, gel would not form at the temperature of ocular cavity so result in rapid ocular clearance of administered drug because it remain in sol form (27). When temperature is increased, the number of vesicles formed increases due to negative coefficient of solubility of niosomes.

Also, the niosomes packed so closely, rendering the solution immobile and thus, the gel is formed. Results revealed that the gelling temperature decreased with increase in the concentration of poloxamer 407 because of the development of larger number of the vesicles that occupy larger volume at lower temperature $(27,28)$. Recently, gelation mechanism on the basis of particle association and interaction has been suggested by several authors. Also, conformation modifications in methyl group orientation in the side chains of poly (oxypropylene) that constitute the core with water removal have also been suggested to contribute to the gelation process (24).

The mucoadhesive agent carbopol 934 NF also causes lowering of gelling temperature. It was due to its property to undergo binding with poly (ethylene) oxide chains present in poloxamer 407 molecule, thus promoting dehydration and cause an increase in entanglement of adjacent molecule with more extensive intermolecular $\mathrm{H}$-bonding and so enhance the association (29). Immediate gelling increased the residence time and improvised the bioavailability of drug. Residence time at the sclera surface is affected by the viscosity of ophthalmic formulation. Hence, viscosity is considered an important parameter for increasing the residence time of ophthalmic formulation upon instillation into cul-de-sac. This property will help in predicting the in vivo behaviour of niosomal dispersion (30). With respect to ocular physiology, the range of shear rate was from 0.03 to $0.14 \mathrm{~s}^{-1}$ during inter blinking period of 4250- $28500 \mathrm{~s}^{-1}$. Thus, the viscosity of formulation should not be such that it may disturb the pseudoplastic behavior of tear film in the eye (30).

From the results, it was found that high viscosity values were observed in all formulations having optimum amount of carbopol $934 \mathrm{NF}$ as it is a viscosity enhancing polymer. So, it helps in increasing the ocular residence time. Percent cumulative drug permeation was found to be less than $60 \%$ because of high amount of poloxamer 407. It was found that presence of poloxamer 407 in gels reduced the release rate of drug. Thus, permeation decreased slightly due to reduction in dimension of water channels. As poloxamer 407 comprises of micelles in aqueous phase in very large amount, the incorporated niosomes may be released by diffusion through gel matrix.

Niosomal permeation may also be affected by the viscosity of gel, aqueous channel size and niosomal distribution. Because of this reason, niosomal gel permeation goes on decreasing from NG1 to NG5. Also, increase in poloxamer cause slight increase in viscosity and decrease in release and thus decrease in the permeation of niosomes was observed. Thus, the minimum permeation showed by NG5 which consists of $20 \%$ w/v poloxamer and maximum permeation showed by NG1 consist of $12 \% \mathrm{w} / \mathrm{v}$ of poloxamer 407. Among all the gels, NG5 was selected as an optimized gel based formulation 
which was then characterized for in vitro mucoadhesion and histological study. The results of in vitro mucoadhesion revealed that variable amount of carbopol $934 \mathrm{NF}$ affected the mucoadhesive strength. The mucoadhesive strength depends on wetting and swelling of carbopol $934 \mathrm{NF}$ that permitted proper contact with scleral tissue. Interaction of carbopol 934NF (bioadhesive in nature) chains with sclera tissue leads to entanglement and weak chemical bonds formed between the entangled chains. Carboxyl group present in carbopol 934 NF exhibits hydrogen bonding which reveals sol to gel transition as the temperature rises above $28^{\circ} \mathrm{C}$. The formation of hydrogen bonding resulting in strengthening the network between polymer and sclera tissue. The results signified the presence of mucoadhesive force to resist the shear force at the time of reflex blinking.

Thus, it can be put into conclusion that the formulation might showed desirable residence in the scleral area. Niosomal based formulation $\mathrm{NF} 2$ showed better release of drug in comparison to marketed preparation because it follows sustained release of drug. This was due to the fact that drug release occurred through niosomes in a controlled manner for a selective time period (31). The release profile obtained defined the greater controlled release ability of developed niosomal in situ gel as compared to NF2. Decrease in drug release from the gel matrix was observed in the niosomal in situ gel in comparison to niosomes and marketed formulation. Finally, proven the fact that marketed preparation did not showed controlled release and did not possessed retaining capacity. Thus, NG5 formulation was found to be best among all three formulation in case of retaining and release manner of drug. Further, results of histological analysis revealed the safety of niosomal in-situ gel via ocular route.

\section{Conclusion}

ATZ- $\beta$-CD complex loaded niosomal in situ gel of (NG5) is depicted to be safe and effective alternative for ocular delivery that overcomes the limitations of conventional therapy. Also minimizes systemic side effects associated with the oral delivery of ATZ. The prepared system proved to have better retention ability and exhibit localized delivery. Thus, it can be put into conclusion that delivery via complex-vesicle based system exhibited potential to carry hydrophobic drug to scleral segment of the eye and capable of treating bacterial conjunctivitis.

\section{Author's contribution}

All the authors have contributed substantially to the design, performance, analysis, or reporting of the work. Dr. Kamla Pathak has contributed in drafting, editing and revising the manuscript. Mr. Rahul Kumar Singh has carried out the bench work, laboratory analysis and Ms. Nida Akhtar has conducted the literature survey, collected the data and drafted the manuscript.

\section{Conflict of interest}

Authors declare no conflict of interest.

\section{References}

1. Lin HH, Ko SM, Hsu LR, Tsai YH. The preparation of norfloxacin loaded liposomes and their in vitro evaluation in pig's eye. J Pharm Pharmacol 1996;48:801-5.

2. Tarabishy AB, Jeng BH. Bacterial conjunctivitis: a review for internists. Cleveland Clinic J Med 2008;75:507-12.

3. Alvarez-Lorenzo C, Yanez F, Barreiro-Iglesisa R, Concheiro A. Imprinted soft contact lenses as norfloxacin delivery systems. J Controlled Release 2006;113:236-44.

4. Grateri T, Gelfuso GM, Freitas O, Rocha EM, Lopez RF. Enhancing and sustaining the topical ocular delivery of fluconazole using chitosan solution and poloxamer/chitosan in situ forming gel. Eur J Pharm Biopharm 2011;79:320-27.

5. Utani CA. Update and critical appraisal of the use of topical azithromycin ophthalmic $1 \%$ solution in the treatment of ocular infections. Clin Ophthalmol 2011;5:801-09.

6. Gilhotra RM, Nagpal K, Mishra DN. Azithromycin novel drug delivery system for ocular application. Int $\mathbf{J}$ Pharm Investig 2011;1:22-8.

7. Kumarasen C. Development of novel ocusert contain norfloxacin and in vitro evaluation. J Pharm Res 2011;4:393-5.

8. Challa R, Ahuja A, Ali J, Khar RK. Cyclodextrins in drug delivery: an updated review. AAPS PharmSciTech 2005;6:32957

9. Kute SD, Sakore SC, Chakraborty BS. Formulation approaches in ocular drug delivery system. Int J Pharm Tech 2010;2:11845 .

10. Akhtar N. Vesicular ocular drug delivery system, preclinical and clinical perspective of drugs delivered via niosomes. Int $\mathrm{J}$ Biopharm 2013;4:38-48.

11. Abdelbary G, El-gendy N. Niosome-encapsulated gentamicin for ophthalmic controlled drug delivery. AAPS PharmSciTech 2008;9:740-7.

12. Liu Z, Li J, Nie S, Liu H, Ding P, Pan W. Study of an alginate/HPMC based in situ gelling ophthalmic delivery system for gatifloxacin. Int J Pharm 2006;315:12-17.

13. Higuchi T, Connors KA. Phase-solubility techniques. Adv Anal Chem Instrum 1965;4:117-210.

14. Qi H, Chen W, Huang C, Li L, Chen C, Li W, et al Development of a poloxamer analogs/carbopol-based in situ gelling and mucoadhesive ophthalmic delivery system for puerarin. Int J Pharm 2007;337:178-87.

15. Jaiswal M, Kumar M, Pathak K. Zero order delivery of itraconazole via polymeric micelles incorporated in situ ocular gel for the management of fungal keratitis. Colloids Surf B Biointerfaces 2015;130:23-30.

16. Loftsson T, Jarto P, Masson M, Jarvinen T. Cyclodextrin in drug delivery. Expert Opin Drug Deliv 2005;2:335-51.

17. Akhtar N, Arkvanshi S, Bhattacharya SS, Verma A, Pathak K. Preparation and evaluation of a buflomedil hydrochloride Niosomal patch for transdermal delivery. J Liposome Res 2015;25:191-201.

18. Gan Q, Wang T. Chitosan nanoparticles as protein delivery carrier systematic examination of fabrication conditions for efficient loading and release. Coll Surf B Biointerf 2007;59:2434.

19. Agnihotri SM, Vavia PR. Diclofenac- loaded biopolymeric nanosuspentions for ophthalmic applications. Nanomedicine 2009;5:90-5.

20. Chhabra G, Chuttani K, Mishra AK, Pathak K. Design and evaluation of nanoemultion drug delivery system of amlodipine besilate for improvement of oral bioavailability. Drug Develop Ind Pharm 2011;37:907-16

21. Pignatello R, Bucolo C, Ferrara P, Maltese A, Puleo A, Puglisi 
G. Eudragit RS100 nanosuspention for the ophthalmic controlled delivery of ibuprofen. Eur J Pharm Sci 2002;16:5361.

22. Shirsand SB, Para MS, Kumar ND, Kanani KM, Keerthy D Formulation and evaluation of ketoconazole niosomal gel drug delivery system. Int J Pharm Investing 2012;2:201-7.

23. Tamizharasi S, Dubey A, Rathi V, Rathi JC. Development and characterization of niosomal drug delivery of gliclazide. J Young Pharm 2009;1:205-9.

24. Uchegbu IF, Florence AT. Non-ionic surfactant vesicles (niosomes): Physical and pharmaceutical chemistry. Adv Coll Interf Sci 1995;58:1-55

25. Maurya SD, Prajapti SK, Gupta AK, Saxena GK, Dhakar RC. Formulation development and evaluation of ethosome of stavudine. Ind J Pharm Edu 2010;44:102-8.

26. Srividya B, Cardoza RM,Amin PD. Sustained ophthalmic delivery of ofloxacin from a $\mathrm{pH}$ triggered in situ gelling system. J Controlled Rel 2001;73:205-11.

27. Singh J, Chhabra G, Pathak K. Development of acetazolamide- loaded, pH-triggered polymeric nanoparticulate in situ gel for sustained ocular delivery: in vitro. ex vivo evaluation and pharmacodynamic study. Drug Dev Ind Pharm 2014;40:122332.

28. Riccia EJ, Lunardi LO, Nanclares DMA, Marchetti JM. Sustained release of lidocane from polaxame 407 gels. Int J Pharm 2005;288:235-44.

29. Majithiya RJ, Ghosh PK, Umerethia ML, Murthy RSR. Thermoreversible-mucoadhesive gel for nasal delivery of sumatriptan. AAPS PharmSciTech 2006;7:80-6.

30. Choy Y, Park J, McCarev B, Edelhauser HF, Prausnitz M. Mucoadhesive microdiscs engineered for ophthalmic drug delivery: Effect of particle geometry and formulation on precorneal residence time. Investig Ophthalmol Visual Sci 2008;49:4808-49.

31. Moghddam SRM, Ahad A, Aqil M, Imam SS, Sultana Y. Formulation and optimization of niosomes for topical diacerein delivery using 3-factor, 3-level Box-Behnken design for the management of psoriasis. Mat Sci Eng C 2016;69:789-97. 